\title{
Thermal Pain Level Estimation Method with Heart Rate and Cerebral Blood Flow
}

\author{
Kohei Arai ${ }^{1}$, Asato Mizoguchi ${ }^{2}$, Hiroshi Okumura ${ }^{3}$ \\ Department of Information Science, Saga University, Saga City, Japan
}

\begin{abstract}
Method for thermal pain level estimation with heart rate and cerebral blood flow using SVM is proposed. Through experiments, it is found that thermal pain level is much sensitive to the cerebral blood flow rather than heart rate. Also, it is found that the performance of thermal pain estimation is much better than the previously proposed method with the number of blinks, the enlarging rate of pupil size.
\end{abstract}

Keywords-Thermal pain; support vector machine; thermal stimulus; classification; cerebral blood flow; heart rate

\section{INTRODUCTION}

A bedridden person, a person who has become a plant state, a person who cannot speak words well. Such people are difficult to convey their feelings. Therefore, there is a strong demand to create methods and systems which allows pain level prediction and representation for disable persons. Life and stress cause blood flow deterioration. In menopausal age, stress and other hormonal disturbances, capillary blood flow is not the movement of most capillaries itself, but blood flow is determined by the expansion of blood vessels called "arterioles" that lead to capillaries. Stress and autonomic nerves are very closely related. The autonomic nerve is one of the peripheral nerves and is a narrow neural network stretched to every corner of the body. It is divided into two, sympathetic and parasympathetic, and extends to all visceral organs such as blood vessels, heart, lungs and intestines. The blood flow in our brain is controlled by various regulatory functions. The typical ones are the carbon dioxide-mediated chemical regulation function in blood and the regulation function by the action of the autonomic nervous system around blood vessels in the brain.

Previously, a method for thermal pain level prediction with human eye motions, in particular, with the number of blinks and the pupil size change rate using SVM is proposed [1]. There are previously proposed methods which use pupil size [2]. It, however, is found that thermal pain level is much sensitive to pupil size change rate than pupil size itself [1]. Such this sensitivity analysis and comparative study is conducted. One of the biggest problems of the previous method is poor accuracy of thermal pain level prediction. It is still not good enough for practical use of the thermal pain level estimation. In order to overcome this situation, a new method with cerebral blood flow and heart rate which are measured by Near Infrared: NIR sensor is proposed here. In particular, thermal pain level measurement with cerebral blood flow is original idea in this study area. Also, it is found that cerebral blood flow is effective to estimate thermal pain level.
The next section describes related research works and the proposed method for prediction of thermal pain level followed by experimental method and results. Then, the results are discussed followed by conclusion. Finally, future research work is described.

\section{Method for Thermal Pain Prediction With Cerebral Blood Flow and Heart RATE BASED ON SVM}

\section{A. Related Research Works}

There are not so small number of methods of thermal pain level prediction such as MEG, MRI, fMRI, EOG, EEG, etc. For instance, Pradkij Panavaranan ; Yodchanan Wongsawat proposed EEG-based pain estimation via fuzzy logic and polynomial kernel support vector machine [3]. They said that the results of pain estimation via fuzzy logic can be roughly indicate the pain state of EEG. The polynomial kernel support vector machine classifier for pain classification has high accuracy.

Also, Ruth Defrin Avi Ohry Nava Blumen Gideon Urca discussed sensory determinants of thermal pain [4].They said that both noxious heat and cold elicited a sensation of heat pain. No consistent pattern of heat-elicited pain was observed in areas in which only cold sensation was intact. Also their data suggest that the integrity of non-noxious thermal systems is essential for the normal perception of thermal pain, and that the subjective sensation of pain depends on the integration of information from nociceptive and non-nociceptive channels.

Furthermore, Raul Fernandez Rojas, Xu Huang \& KengLiang $\mathrm{Ou}$ proposed a machine learning approach for the identification of a biomarker of human pain using fNIRS [5]. They said that feature extraction was completed in three different domains (time, frequency, and wavelet), and a total of 69 features were obtained. Feature selection was carried out according to three criteria, information gain (IG), joint mutual information (JMI), and Chi-squared $(\chi 2)$. The significance of each feature ranking was evaluated using three learning models separately, linear discriminant analysis (LDA), the K-nearest neighbor (K-NN) and support vector machines (SVM) using the linear and Gaussian and polynomial kernels. The results showed that the Gaussian SVM presented the highest accuracy (94.17\%) using only 25 features to identify the four types of pain in our database. In addition, we propose the use of the top 13 features according to the JMI criteria, which exhibited an accuracy of $89.44 \%$, as promising biomarker of pain. This study contributes to the idea of developing an objective assessment of pain and proposes a potential biomarker of human pain using fNIRS. 
James C. Eisenach, Regina Curry, Carol A. Aschenbrenner, Robert C. Coghill Timothy, T.Houle investigated pupil responses and pain ratings to heat stimuli in particular, for a reliability and effects of expectations and a conditioning pain stimulus [6]. These, however, are not so easy to measure and prediction of thermal pain due to the fact that it is difficult to recognize thermal pain with measured data and also it does cost for the measuring instruments. Also, these are not accurate enough (around $75 \%$ of accuracy with some time delay) [6].

Pupillary responses to thermal pain stimulation in healthy volunteers (Lauren A. Banker) ${ }^{1}$. Pupil responses and pain ratings to heat stimuli: Reliability and effects of expectations and a conditioning pain stimulus (James C. Eisenach et al.) [6]. Although these studies are based on only the pupillary response, in this research I add elements of other eye motion.

Justin E. Brown, Neil Chatterjee, Jarred Younger, Sean Mackey investigated a physiology-based measure of pain: patterns of human brain activity distinguish painful from nonpainful thermal stimulation [7]. Although this study is a study of brain activity by fMRI data using classification method of Support Vector Machine: SVM, this research is a study using eye motion data using SVM.

\section{B. Previous System Configuration}

Near Infrared: NIR camera which is mounted on a glass is used for acquisition of eye images. Fig. 1 shows the outlook and schematic view of the previously proposed system.

NIR camera imagery data is acquired through USB interface to PC. The outlook of the camera is shown in Fig. 2 while major specification of the camera is shown in Table I.

The experiment we conducted this time was HOT 121B developed by Hitachi. Heat stimulation is intended to be measured using the Near Infrared Sensor: NIRS which is primarily measures brain activity. The cerebral blood flow and heart rate or pulse can be measured when given and examine the change. Outlook of the HOT 121B is shown in Fig. 4 while close-up photo of the NIRS sensors are shown in Fig. 5, respectively. The cerebral blood volume of the prefrontal cortex in Alzheimer type dementia patients and healthy elderly people is measured using the near-infrared light measurement device (HOT121B), the data is corrected, and the corrected data and The data was compared and examined to clarify the difference between the two.

\section{Proposed System Configuration}

The system configuration of the proposed thermal pain estimation system is shown in Fig. 3.

Meanwhile, the heat source (thermal stimulus) is shown in Fig. 6. In addition, based on the data obtained by measurement, identification of measurement results was also performed using SVM (support vector machine) ${ }^{2}$. SVM is well known classification method which allows classify the data into the previously designated class categories. In the proposed method, two class categories, pain and not pain are designated. By using the feature components, SVM based classification is applied.

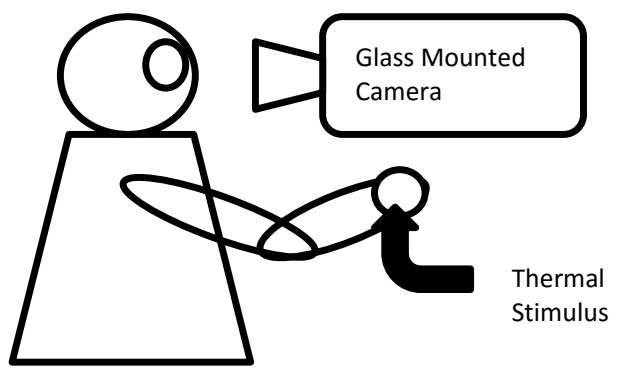

(a)Schematic view of the previous system

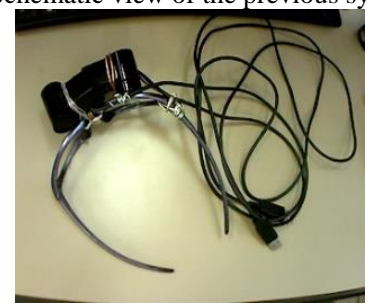

(b)Outlook

Fig. 1. Outlook and Schematic View of the Previously Proposed System Configuration.

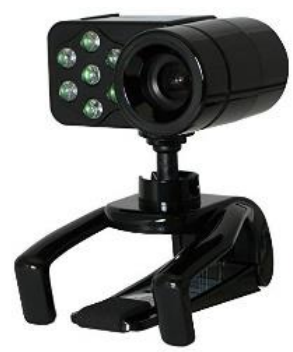

Fig. 2. Outlook of the NIR Camera used.

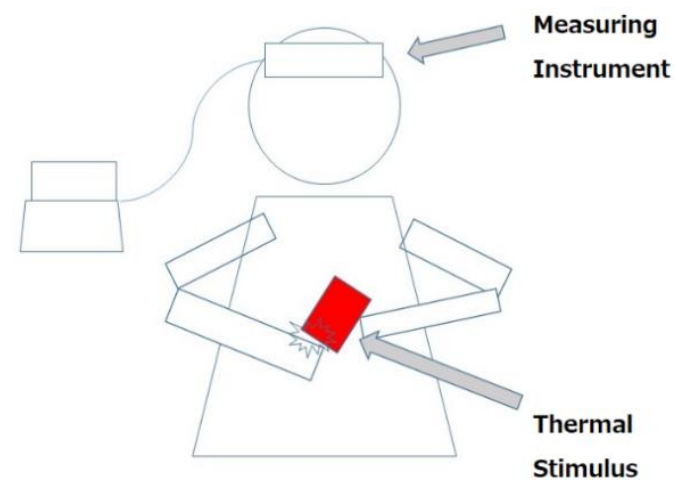

Fig. 3. System Configuration of the Proposed Thermal Pain Estimation System with NIRS Sensors.

TABLE. I. MAJOR SPECIFICATION OF THE NIR CAMERA USED

\begin{tabular}{|l|l|}
\hline weight & $200 \mathrm{~g}$ \\
\hline Size(dimension) & $21.8 \times 14.8 \times 7.6 \mathrm{~cm}$ \\
\hline Camera Name & DC-NCR13U \\
\hline
\end{tabular}

${ }^{1}$ https://steinhardt.nyu.edu/appsych/psych_cafe

${ }^{2}$ http://opencv.jp/sample/svm.html 


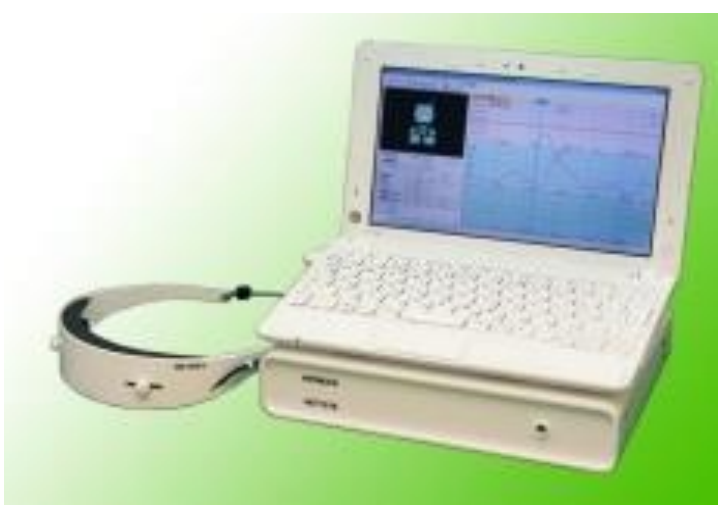

Fig. 4. Outlook of the HOT 121B.

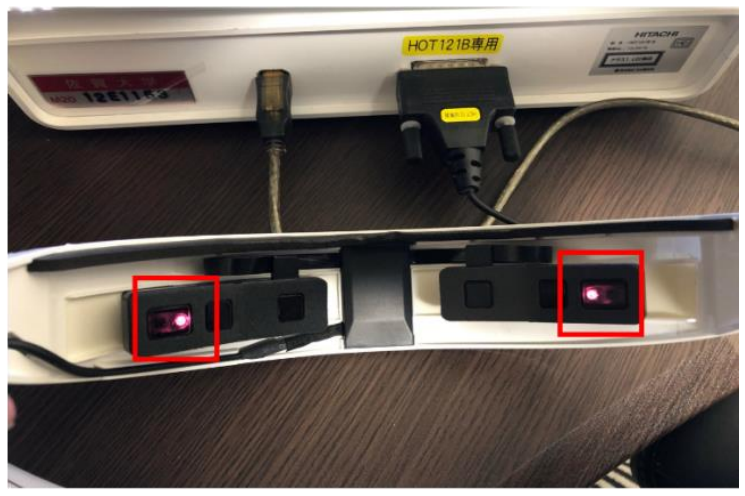

Fig. 5. Close-up Photo of the NIRS Sensors of the HOT 121B.

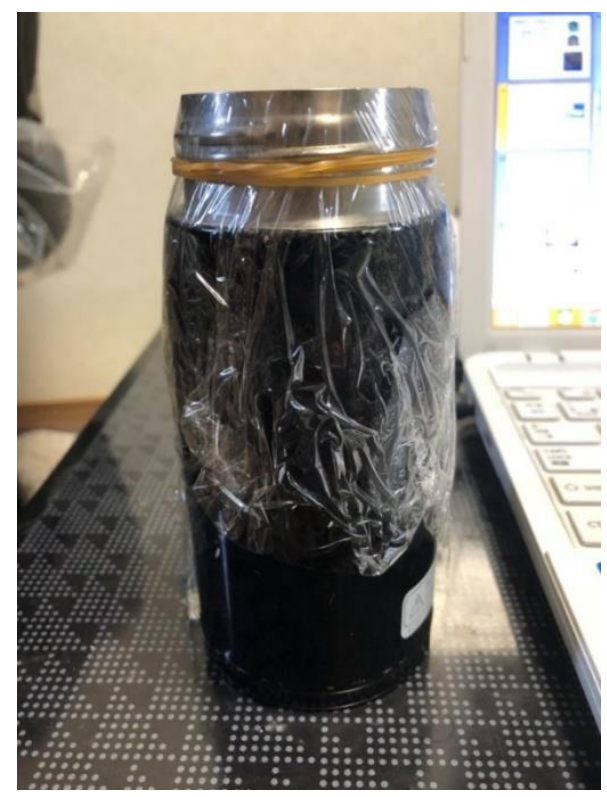

Fig. 6. Outlook of the Thermal Stimulus.

\section{EXPERIMENTS}

\section{A. Experimental Configuration}

A heat-retaining bottle was covered with a wrap, and a rubber band was attached, and hot water was put in it to secure a heat source. Start the measurement with the cerebral blood flow measurement device, repeat the action of putting the heat source on the palm 10 seconds for 60 seconds and repeating the measurement three times for 210 seconds and prepare two kinds of temperature of 42 degrees and 53 degrees, and experiment went through as shown in Fig. 7.

Example of the acquired data of cerebral blood (left and right), heart rate (left and right), LF/HF (left and right) and thermal stress (Temp.) is shown in Fig. 8(a) and (b). The HF and LF of autonomic nervous function activity as stress indicators are as follows. HF is an abbreviation for High Frequency and is a fluctuating wave that uses respiration with a period of 3 to 4 seconds as a signal source. Or it is the sum of the power spectrum of the frequency domain. Also, LF is a component of lower frequency than that. HF, LF components and points to be noted when used HF components are generally calculated as the sum of power spectrum components of 0.15 to $0.40 \mathrm{~Hz}$, and LF components are generally calculated as the sum of power spectrum components of 0.04 to $0.15 \mathrm{~Hz}$. is there.

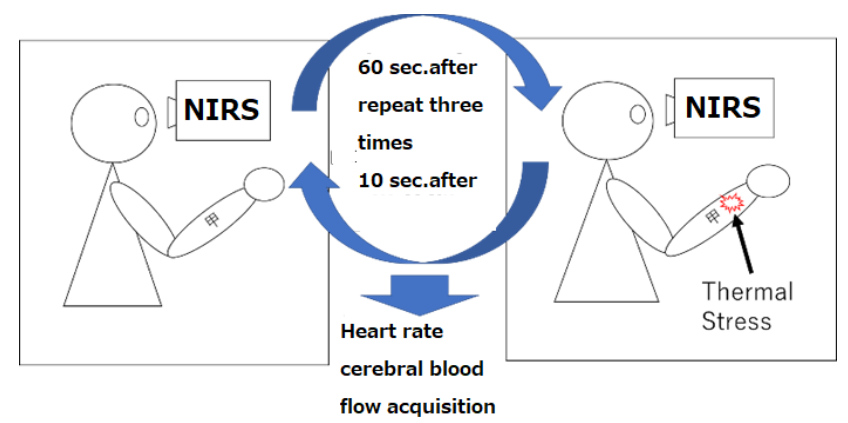

Fig. 7. Experimental Procedure.

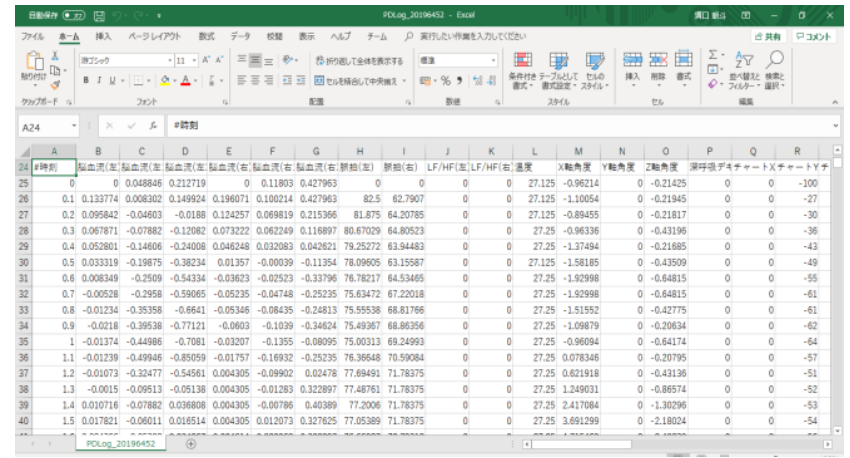

(a) Spread sheet

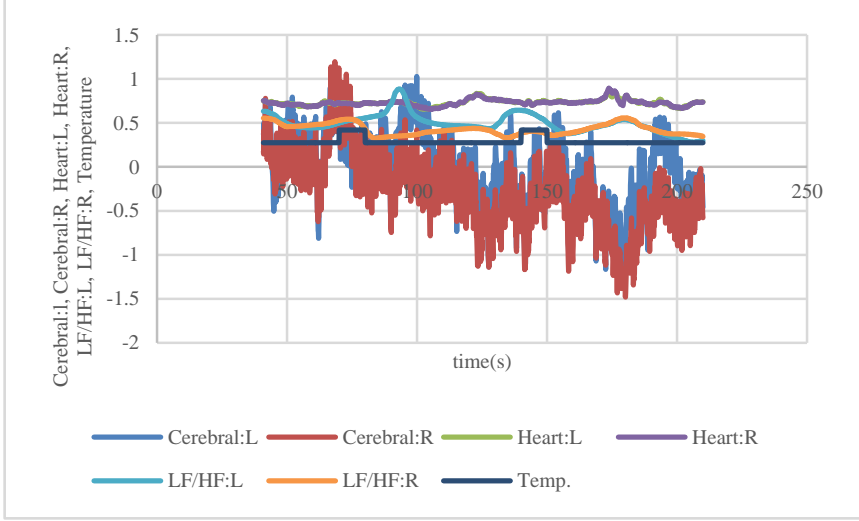

(b) Graph

Fig. 8. Example of the Acquired Data of Cerebral Blood (Left and Right), Heart rate (Left and Right), LF/HF (Left and Right) and Thermal Stress (Temp). 
The values divided by the sympathetic nerve (LF) and parasympathetic nerve (HF) figures do not always match the figures indicated by the balance (LF / HF). It is necessary to take into account the strength of the autonomic nervous system (the age of the autonomic nervous system) and the like as a view of the autonomic nervous system evaluation (overall evaluation).

The acquired LF/HF data means the frequency component ratio between low frequency and high frequency components of cerebral blood flow. These data are closely related psychological status between Parasympathetic nervous system and Sympathetic. Although it is useful for thermal stress detection, just cerebral blood flow and heart rate are focused in this study.

\section{B. Experimental Results}

Fig. 9(a) shows the acquired time series of the cerebral blood flow for the thermal stimulus of 42 degree $\mathrm{C}$ while Fig. 9(b) shows that for 53 degree C, respectively. Meanwhile, Fig. 9(c) shows the acquired time series of the heart rate for 42 degree $\mathrm{C}$ of the thermal stimulus wile Fig. 9(d) shows that for 53 degree $\mathrm{C}$, respectively.

On the other hand, Fig. 10(a) shows the comparison of cerebral blood flow between thermal stimulus of 42 degree $\mathrm{C}$ and 53 degree $\mathrm{C}$ while Fig. 10(b) shows that for heart rate. From these figures, it is understandable that it is possible to estimate the thermal stimulus temperature using cerebral blood flow and heart rate.

\section{Machine Learning with SVM for Estimation of Thermal Stress of Temperature}

Using these data, machine learning process is applied for estimation of thermal stress temperature based on SVM. The number of dataset of cerebral blood flow and heart rate is 20 for learning process. Table II(a) shows the results of the learning process for heart rate while Table II(b) shows those for cerebral blood flow. Percent Correct Classification: PCC for heart rate is 0.835 while that for cerebral blood flow is 0.898 . In the previously proposed thermal stress detection by using size of pupil and pupil size changing rate, accuracy is around 0.8 . Therefore, it is found that the proposed method for thermal stress temperature estimation is better that the previously proposed method with pupil related data.

In this experiment, only two thermal stress temperature were tested. Therefore, there is an applicable limitation of the proposed method (up to 53 degree $\mathrm{C}$ of temperature). It is not clear that cerebral blood flow response against much higher water temperature of thermal stress and heart rate. Therefore, dynamic rage of the thermal stress temperature has to be expended for the next step of additional experiments. Furthermore, it might be exist some nonlinearity of the relation between cerebral blood flow and thermal stress temperature. Therefore, another experiment has to be conducted with much higher thermal stress temperature. These are applicable limitations of the proposed method.

\section{Cerebral blood flow ( $\mathrm{mM} / \mathrm{mm})$}

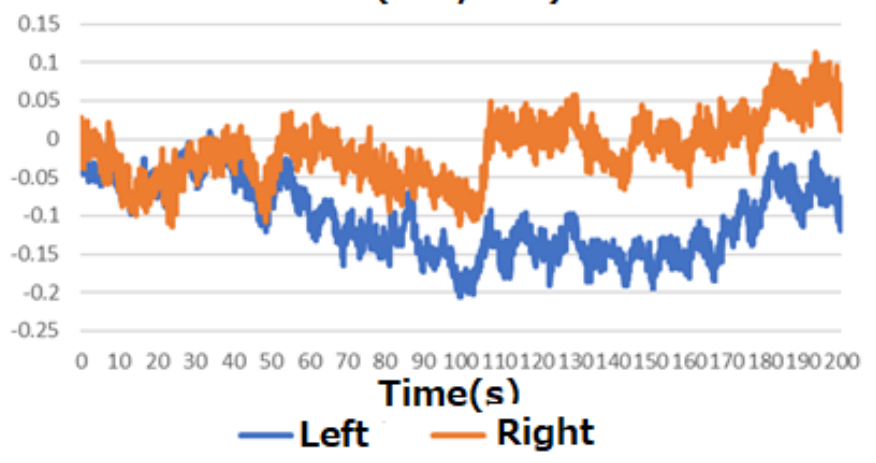

(a) 42 degree $\mathrm{C}$

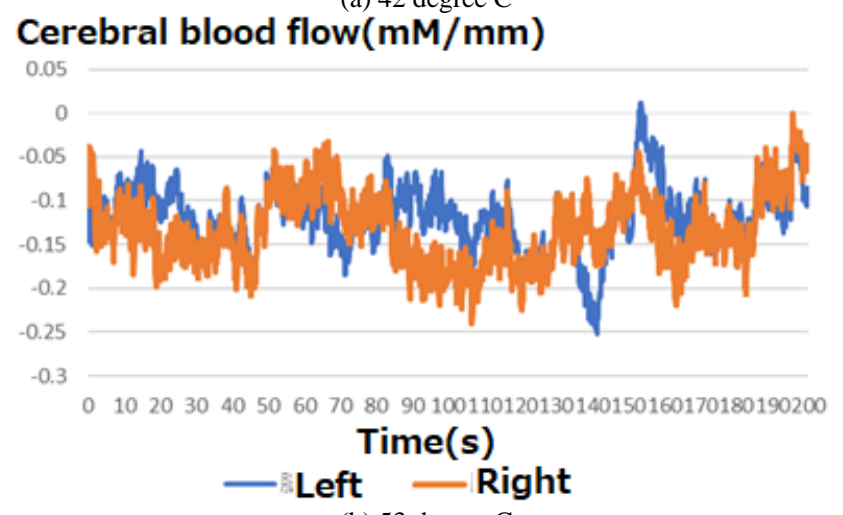

Heart Rate

(b) 53 degree $\mathrm{C}$

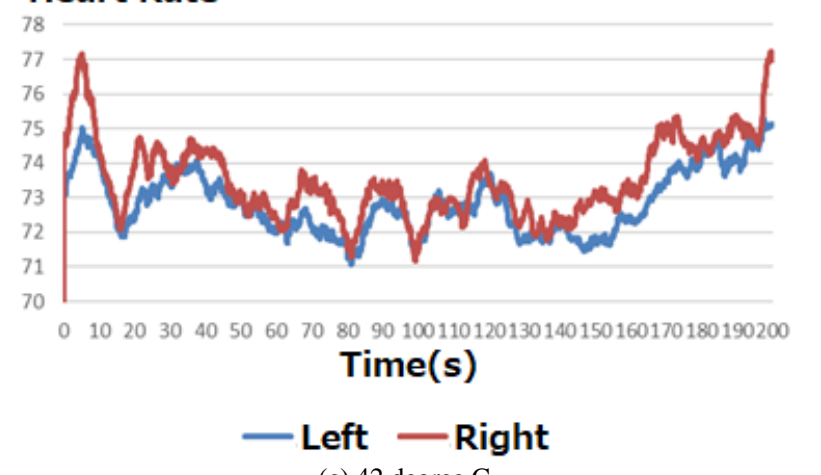

Heart Rate

(c) 42 degree $\mathrm{C}$

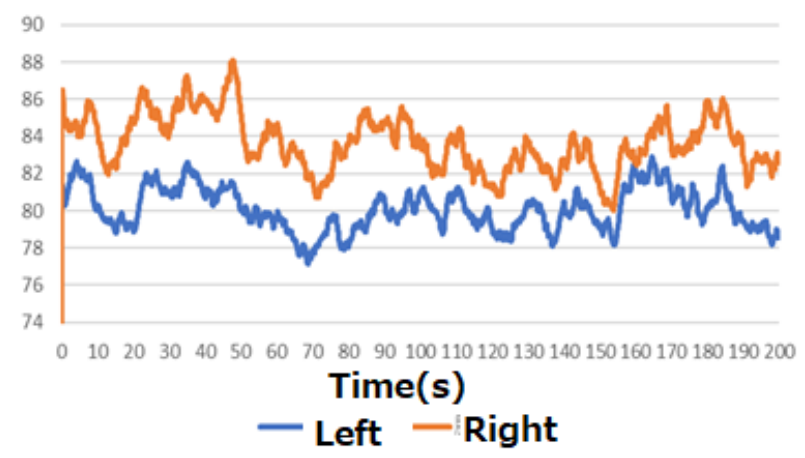

(d) 53 degree $\mathrm{C}$

Fig. 9. The Acquired Cerebral Blood Flow and Heart Rate. 


\section{Cerebral blood flow $(\mathrm{mM} / \mathrm{mm})$}

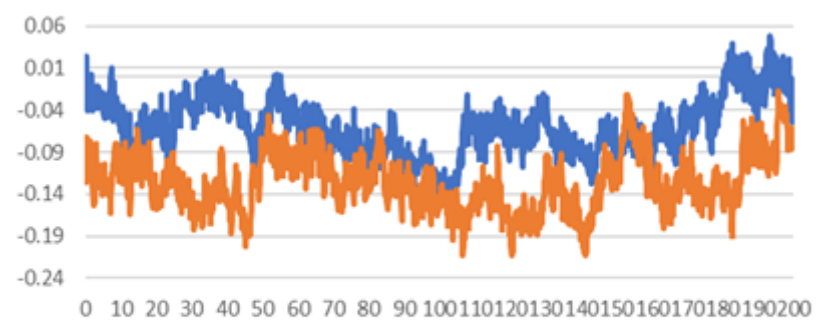

Time(s)

\section{$-42 \mathrm{deg} . \mathrm{C}-53 \mathrm{deg} . \mathrm{C}$}

(a) Cerebral Blood Flow.

\section{Heart Rate}

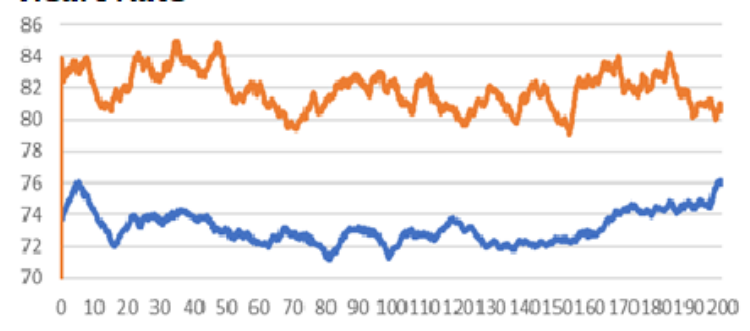

Time(s)

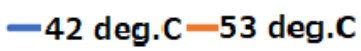

(b) Heart Rate.

Fig. 10. Comparison of cerebral blood flow and heart rate between thermal stimulus of 42 degree $\mathrm{C}$ and 53 degree $\mathrm{C}$.

TABLE. II. RESULTS FROM THE SVM BASED LEARNING PROCESS FOR ESTIMATION OF THERMAL STRESS TEMPERATURE USING HEART RATE AND Cerebral BloOd Flow

(a) Heart Rate

\begin{tabular}{|l|l|l|}
\hline Heart Rate & 42 degree C & 53 degree C \\
\hline 42 degree C & 0.821 & 0.152 \\
\hline 53 degree C & 0.179 & 0.849 \\
\hline
\end{tabular}

(b) Cerebral Blood Flow

\begin{tabular}{|l|l|l|}
\hline Cerebral Blood Flow & 42 degree C & 53 degree C \\
\hline 42 degree C & 0.894 & 0.098 \\
\hline 53 degree C & 0.106 & 0.902 \\
\hline
\end{tabular}

\section{CONCLUSION}

Method for thermal pain level estimation with heart rate and cerebral blood flow using SVM is proposed. Through experiments, it is found that thermal pain level is much sensitive to the cerebral blood flow rather than heart rate. Also, it is found that the performance of thermal pain estimation is much better than the previously proposed method with the number of blinks, the enlarging rate of pupil size.

As the results, it is found that Percent Correct Classification: PCC for heart rate is 0.835 while that for cerebral blood flow is 0.898 . In the previously proposed thermal stress detection by using size of pupil and pupil size changing rate, accuracy is around 0.8. Therefore, it is also found that the proposed method for thermal stress temperature estimation is better that the previously proposed method with pupil related data.

\section{FUTURE WORK}

Further study is required for more experimental data with a variety of examiners. In order to validate the proposed method, more experiments with a variety of examiners are highly required. In particular, the other influencing factors to the thermal pain level changes such as pupil size eye motion, the number of blinks, the enlarging rate of pupil size. etc. have to be added as features of the SVM classification for improvement of the thermal pain level estimation accuracy.

\section{ACKNOWLEDGMENT}

The author would like to thank Prof. Dr. Osamu Fukuda of Saga University for his comments and suggestions throughout this study.

\section{REFERENCES}

[1] Kohei Arai, Method for Thermal Pain Level Prediction with Eye Motion using SVM, International journal of Advanced Computer Science and Applications: IJACSA, 9, 4, 170-175, 2018.

[2] D. Alnaes, M.H. Sneve, T. Espeseth, T. Endestad, S.H. van de Pavert, B. Laeng, Pupil size signals mental effort deployed during multiple object tracking and predicts brain activity in the dorsal attention network and the locus coeruleus, Journal of Visual., 14, 1-20, 2014.

[3] Pradkij Panavaranan ; Yodchanan Wongsawat proposed EEG-based pain estimation via fuzzy logic and polynomial kernel support vector machine The 6th 2013 Biomedical Engineering International Conference, 2013.

[4] Ruth Defrin Avi Ohry Nava Blumen Gideon Urca discussed sensory determinants of thermal pai, Brain, Volume 125, Issue 3, March 2002, Pages 501-510, 2002.

[5] Raul Fernandez Rojas, Xu Huang \& Keng-Liang Ou proposed a machine learning approach for the identification of a biomarker of human pain using fNIRS Scientific Reports 9, Article number: 5645 (2019).

[6] James C. Eisenach, Regina Curry, Carol A. Aschenbrenner, Robert C. Coghill Timothy, T.Houle, Pupil responses and pain ratings to heat stimuli: Reliability and effects of expectations and a conditioning pain stimulus, Journal of Neuroscience Method, 279, 1, 52-59, 2017.

[7] Justin E. Brown, Neil Chatterjee, Jarred Younger, Sean Mackey, Towards a Physiology-Based Measure of Pain: Patterns of Human Brain Activity Distinguish Painful from Non-Painful Thermal Stimulation, PLoS ONE 6(9): e24124, 2011.

\section{AUTHOR's PROFILE}

Kohei Arai, He received BS, MS and PhD degrees in 1972, 1974 and 1982, respectively. He was with The Institute for Industrial Science and Technology of the University of Tokyo from April 1974 to December 1978 also was with National Space Development Agency of Japan from January, 1979 to March, 1990. During from 1985 to 1987, he was with Canada Centre for Remote Sensing as a Post Doctoral Fellow of National Science and Engineering Research Council of Canada. He moved to Saga University as a Professor in Department of Information Science on April 1990. He was a councilor for the Aeronautics and Space related to the Technology Committee of the Ministry of Science and Technology during from 1998 to 2000. He was a councilor of Saga University for 2002 and 2003. He also was an executive councilor for the Remote Sensing Society of Japan for 2003 to 2005 . He is an Adjunct Professor of University of Arizona, USA since 1998. He also is Vice Chairman of the Science Commission "A" of ICSU/COSPAR since 2008 then he is now award committee member of ICSU/COSPAR. He wrote 37 books and published 570 journal papers. He received 30 of awards including ICSU/COSPAR Vikram Sarabhai Medal in 2016, and Science award of Ministry of Mister of Education of Japan in 2015. He is now Editor-in-Chief of IJACSA and IJISA. http://teagis.ip.is.saga-u.ac.jp/index.ht. 